\title{
Local Convertibility and the Quantum Simulation of Edge States in Many-Body Systems
}

\author{
Fabio Franchini, ${ }^{1,2, *}$ Jian Cui, ${ }^{3,4}$ Luigi Amico, ${ }^{5,6}$ Heng Fan, ${ }^{3, \dagger}$ Mile Gu, ${ }^{7,6}$ \\ Vladimir Korepin, ${ }^{8,}$ Leong Chuan Kwek, ${ }^{6,9}$ and Vlatko Vedral ${ }^{10,6}$ \\ ${ }^{1}$ Department of Physics, Massachusetts Institute of Technology, Cambridge, Massachusetts 02139, USA \\ ${ }^{2}$ SISSA and I.N.F.N., Via Bonomea 265, 34136 Trieste, Italy \\ ${ }^{3}$ Institute of Physics, Chinese Academy of Sciences, Beijing 100190, China \\ ${ }^{4}$ Freiburg Institute for Advanced Studies, Albert Ludwigs University of Freiburg, \\ Albertstraße 19, 79104 Freiburg, Germany \\ ${ }^{5}$ CNR-MATIS-IMM \& Dipartimento di Fisica e Astronomia, Via S. Soa 64, 95127 Catania, Italy \\ ${ }^{6}$ Centre for Quantum Technologies, National University of Singapore, \\ 3 Science Drive 2, 117543 Singapore, Singapore \\ ${ }^{7}$ Center for Quantum Information, Institute for Interdisciplinary Information Sciences, \\ Tsinghua University, Beijing 100084, China \\ ${ }^{8}$ C. N. Yang Institute for Theoretical Physics, Stony Brook University, Stony Brook, New York 11794, USA \\ ${ }^{9}$ National Institute of Education and Institute of Advanced Studies, Nanyang Technological University, \\ 1 Nanyang Walk, 637616 Singapore, Singapore \\ ${ }^{10}$ Atomic and Laser Physics, Clarendon Laboratory, University of Oxford, \\ Parks Road, Oxford OX13PU, United Kingdom
}

(Received 19 March 2014; revised manuscript received 2 July 2014; published 13 November 2014)

\begin{abstract}
In some many-body systems, certain ground-state entanglement (Rényi) entropies increase even as the correlation length decreases. This entanglement nonmonotonicity is a potential indicator of nonclassicality. In this work, we demonstrate that such a phenomenon, known as lack of local convertibility, is due to the edge-state (de)construction occurring in the system. To this end, we employ the example of the Ising chain, displaying an order-disorder quantum phase transition. Employing both analytical and numerical methods, we compute entanglement entropies for various system bipartitions $(A \mid B)$ and consider ground states with and without Majorana edge states. We find that the thermal ground states, enjoying the Hamiltonian symmetries, show lack of local convertibility if either $A$ or $B$ is smaller than, or of the order of, the correlation length. In contrast, the ordered (symmetry-breaking) ground state is always locally convertible. The edge-state behavior explains all these results and could disclose a paradigm to understand local convertibility in other quantum phases of matter. The connection we establish between convertibility and nonlocal, quantum correlations provides a clear criterion of which features a universal quantum simulator should possess to outperform a classical machine.
\end{abstract}

DOI: 10.1103/PhysRevX.4.041028

\section{INTRODUCTION}

In 1982, Richard Feynman conjectured that a quantum machine is necessary to predict the outcome of a general quantum evolution and pioneered the notion of a universal quantum simulator: a device capable of processing quantum information that potentially supersedes any classical computer in simulating quantum systems. This idea embraces much of quantum information research and the

\footnotetext{
*abiof@mit.edu

†hfan@iphy.ac.cn

*korepin@gmail.com
}

Published by the American Physical Society under the terms of the Creative Commons Attribution 3.0 License. Further distribution of this work must maintain attribution to the author(s) and the published article's title, journal citation, and DOI.
Subject Areas: Condensed Matter Physics, Quantum Physics, Quantum Information

technologies stemming from it $[1,2]$ and has attracted a lot of efforts toward the realization of such a device. However, quantifying to what extent a given quantum system could outperform a classical simulator is problematic [3]. How can we determine if a many-body system can operate as an efficient quantum simulator? To what extent is coherent manipulation the defining property of a quantum algorithm? We address such a question quantitatively, using the local convertibility of the quantum system hosting the simulation, and we demonstrate that the (Majorana) edge states establish genuinely quantum long-range correlations that may provide an additional resource for a given computational protocol.

While quantum complexity and quantum algorithm theory have provided very general results on the computational power of abstract models [4-6], specific toy models (often providing concrete and physically relevant physical 
models) afford instances that exhibit interesting behavior in their own right (see, for instance, Refs. [7,8]). In the present work, we follow the latter avenue. Notwithstanding that quantum many-body systems provide a natural setting for entanglement and other quantum superposition or interference effects [9-15], it is poorly understood which one of the quantum resources can indeed play as the added value for the simulation [3,16-24]. On the other hand, an important achievement has been the identification of the role of short- and long-range entanglement, which have also emerged as figures of merit for the different quantum orders that can be established in extended systems [25].

Here, we refer to a specific notion of long-range entanglement related to local operations and classical communications (LOCC) [26]. Upon partitioning a many-body spin system into two blocks $A$ and $B$, we consider the following question: Can the response of the ground state $|g\rangle$ to an external perturbation be rendered through LOCC restricted to $A$ and $B$ individually? If affirmative, the ground state can be moved around within a given quantum phase by LOCC. If negative, the adiabatic evolution induced by the perturbation involves some coherent quantum operation between system $A$ and system $B$. The figure of merit for such phenomena is the differential local convertibility (DLC) of bipartite states. DLC was introduced in the context of majorization [27-29]. Quantitatively, DLC accounts for the response of the Rényi entropy

$$
S_{\alpha} \doteq \frac{1}{1-\alpha} \log \operatorname{Tr} \rho_{A}^{\alpha}
$$

to the changing of a control parameter $h$ in the Hamiltonian. Here, $\rho_{A} \doteq \operatorname{Tr}_{B}|g\rangle\langle g|$ is the reduced density matrix of the block $A$ and $\alpha$ is a free parameter that tunes different entanglement measures [26]. For instance, while low $\alpha$ 's weight more evenly all eigenvalues of $\rho_{A}$, higher values of $\alpha$ enhance the role of the larger eigenvalues, which, as we are going to show, are more sensitive to the edge-state behavior.

DLC holds if and only if all the $\alpha$ entropies are monotonous:

$$
\partial_{h} S_{\alpha} \geq 0, \quad \forall \alpha \geq 0
$$

or $\partial_{h} S_{\alpha} \leq 0, \forall \alpha \geq 0$ [30,31]. DLC was first employed in a many-body problem by Cui et al. in Ref. [32]. An important motivation behind Ref. [32] is the observation that an adiabatic quantum algorithm [33] may exhibit greater computational capabilities only in a given phase that is not DLC. Here, by identifying a physical mechanism responsible for lack of DLC, we argue that the computational power of a nonconvertible phase is actually much bigger and that a quantum phase cannot be exploited as an efficient (universal) quantum simulator [1] if it is locally convertible.
We explain the phenomenology of local convertibility in quantum phases supporting edge states (that is, excitations localized at the boundaries of a many-body system) $[34,35]$. We shall show that in phases with boundary states, the operation of dividing the system into two partitions reveals long-ranged correlations that cannot be resolved within the partition. These correlations are the manifestation of the edge states created at the boundaries between the subregions. These considerations are reflected by the nontrivial behavior of the entanglement entropy: For some (low) $\alpha$ 's, the Rényi entropies are sensitive to short-range entanglement and increase when a quantum phase transition (QPT) is approached, but for other (large) $\alpha$ 's, the entropies do the opposite. This finding implies that the entanglement between edge states can decrease, even as the correlation length increases.

\section{THE QUANTUM ISING CHAIN}

To show the effect of edge states on local convertibility, it is desirable to have a model with three properties: (i) It should support edge states, (ii) quasiparticle excitations should be clearly identifiable, and (iii) there should be a mechanism for destroying the edge states and observing the different behavior. The one-dimensional transverse-field Ising model fulfills these requirements [36,37]. It is defined by the Hamiltonian

$$
H_{I}=-\sum_{j=1}^{N}\left(t \sigma_{j}^{x} \sigma_{j+1}^{x}+h \sigma_{j}^{z}\right),
$$

where $\sigma_{j}^{\alpha}$ are Pauli matrices, $t$ is a hopping amplitude (which we can set to $t=1$ ), and $h$ is the control parameter for the external magnetic field. A quantum phase transition for $h=t=1$ happens in the thermodynamic limit of $N \rightarrow \infty$. This QPT's signatures have recently been observed experimentally [38]. We note that with an additional $\sigma_{z} \sigma_{z}$ and by allowing a distribution of the couplings on a sparse graph, the Hamiltonian (3) would result in a quantum Merlin-Arthur (QMA) hard ground-state problem, hence providing a universal quantum simulator [39]. It has also been shown in Ref. [39] that with all off-diagonal matrix elements in the standard basis being real and sharing the same sign, the model falls in the class of so-called stoquastic systems; see Refs. [39,40] for related topics.

The Hilbert space acted on by the Hamiltonian (3) can be described in terms of eigenstates of the string operator $\mu_{N}^{x}=\prod_{j=1}^{N} \sigma_{j}^{z}$, which generates the $\mathbb{Z}_{2}$ symmetry of Eq. (3). For $h>1$, the system is paramagnetic with $\left\langle\sigma^{x}\right\rangle=0$. For $h<1$, the spectrum of the Ising model becomes doubly degenerate. A ground state that is also an eigenstate of $\mu_{N}^{x}$ has a vanishing order parameter $\left\langle\sigma^{x}\right\rangle=0$. This ground state is known as the "thermal ground state." This state is also the initial state employed in the 2SAT problem and in adiabatic quantum computation protocols for finite $N$ [33]. In the thermodynamic limit $(N \rightarrow \infty), \sigma^{x}$ can acquire a nonzero 
expectation value. The symmetry will be broken spontaneously, and the ground state will be given by the (anti) symmetric combination of the two eigenstates of $\mu_{N}^{x}$. For $h<1$, we consider both the ferromagnetic ground state with nonvanishing order parameter $\left\langle\sigma^{x}\right\rangle$ and the thermal one enjoying the same $\mathbb{Z}_{2}$ symmetry as the Hamiltonian.

The quantum Ising model (3) can be mapped exactly, although nonlocally, to a system of free spinless fermions $\left\{c_{j}, c_{j}^{\dagger}\right\}$ [36]. We remark that the mapping in Ref. [36] preserves the entanglement between $A$ and $B[41,42]$ and generates the Kitaev chain. As emphasized in Ref. [43], this formulation highlights the presence of Majorana edge states as emergent degrees of freedom. Majorana fermions are the elusive particles (coinciding with their own antiparticles), proposed by Ettore Majorana. Many research groups are trying to find and manipulate them [34,35]. Each Dirac fermion of the chain can be used to define two Majorana fermions:

$$
\begin{aligned}
f_{j}^{(1)} & \equiv\left[\prod_{l<j} \sigma_{l}^{z}\right] \sigma_{j}^{x}=c_{j}^{\dagger}+c_{j}, \\
f_{j}^{(2)} & \equiv\left[\prod_{l<j} \sigma_{l}^{z}\right] \sigma_{j}^{y}=i\left(c_{j}^{\dagger}-c_{j}\right) .
\end{aligned}
$$

We represent this mapping pictorially in Fig. 1. In the paramagnetic phase $(h>1)$, the Hamiltonian pairs predominantly Majoranas on the same site $j$. (This correlation is drawn as a double line in the picture.) In the ferromagnetic phase $(h<1)$, the dashed line connecting different sites is dominant. In Kitaev's approach, the double degeneracy of this phase emerges as the first and last Majoranas are left unpaired and can be combined into a complex fermion. (The occupancy or vacancy of this fermion costs

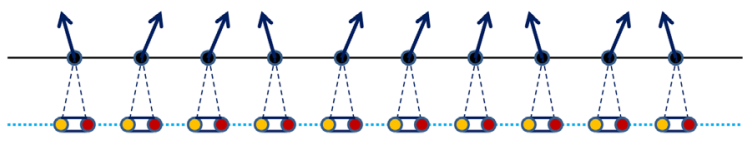

○ Majorana fermion $f^{(1)}$ - Majorana fermion $f^{(2)} \Subset h \quad \bullet \cdots \circ t$

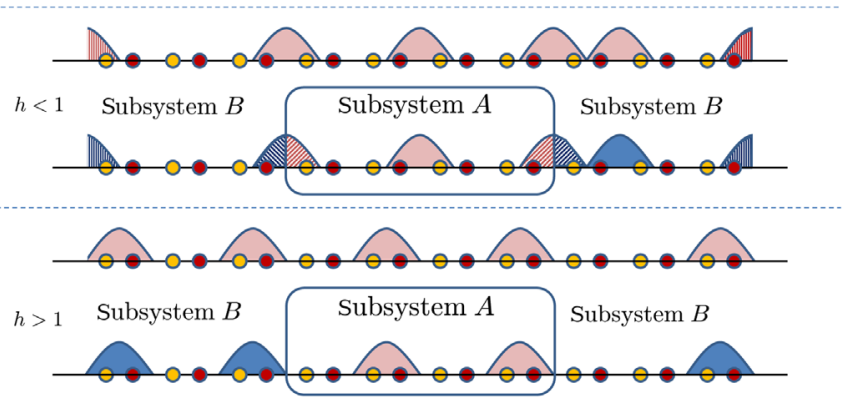

FIG. 1. Top: The Ising chain is mapped into a system of Majorana fermions by doubling the lattice sites. Center and bottom: A schematic of the quasiparticle excitations in the two phases and the effect of bipartitioning the system; for small $h$, edge states form at the opposite boundaries of subsystem $A$. The property of local convertibility depends on the correlations between such edge states. no energy.) We will see that the same picture applies when the system is divided into two partitions: In the ferromagnetic phase, this operation cuts the dominant link and leaves unpaired Majorana edge states on each side of the cut.

This behavior is a key many-body feature that renders phases supporting boundary states more "quantum" than other systems. In fact, since any subsystem develops edge states, in these phases, qubits of information are stored nonlocally between the sites and we will see that this property is mirrored by the nontrivial entanglement behavior, yielding lack of local convertibility. Such phenomenology must be a necessary ingredient of a machine aimed at simulating a generic quantum system, and this observation is the reason for which we believe nonlocal convertibility to be a strong indicator of a higher computational power.

\section{THE $\mathbb{Z}_{2}$-SYMMETRIC GROUND STATE}

As explained in the Appendix, in the Ising chain, the $2^{L}$ states within a block of $L$ consecutive sites can be constructed in terms of individual quasiparticle excitations, which can be either occupied or empty. These excitations are, in general, delocalized, with a typical size set by the correlation length. However, a $\mathbb{Z}_{2}$-symmetric state possesses one special excitation, with support lying at the opposite edges of the block and formed by two Majorana edge states [43]. When the block is extended to the whole system $(L=N)$, the block excitations coincide with the systems' excitations, including the boundary states.

The entanglement between two subsystems $A$ and $B$ can be extracted from the $2 L$ eigenvalues $\pm i \nu_{j}$ of the correlation matrix equation (A6) incorporating the correlations of the excitations within the spin block. Here, $L$ is the number of lattice sites in $A$. The eigenvalues of the reduced density matrix can then be constructed out of the $\nu_{j}$ 's, using Eq. (A4) in the Appendix. The $\nu$ 's can be interpreted as sort of occupation numbers, since they capture the overlaps between each block quasiparticle excitation and the ground state, according to Eq. (A3): $\nu_{j}=0$ means that this block excitation is half filled and half empty in the ground state, while $\nu_{j}=1$ indicates that the excitation is either completely occupied or not present at all.

In Fig. 2, we plot these eigenvalues $\nu_{j}$ as a function of the magnetic field for $L=2$ and $L=10$. Notice that in both cases, only one block excitation has a nontrivial behavior while the other eigenvalues stay approximately constant around unity in all phases. Significant deviations happen only close to the QPT (as the correlation length diverges). As discussed, the modes with $\nu_{j} \simeq 1$ define bulk states. In contrast, the nontrivial eigenvalue is close to 0 for $h \simeq 0$ and rises rapidly toward 1 crossing the QPT at $h=1$ : In the ferromagnetic phase, it corresponds to a block excitation that is neither occupied nor empty. By cutting the chain into two subregions, we sever the dominant intersite correlation and hence generate two unpaired Majorana edge states (see Fig. 1). We notice, however, that as $h$ increases, the 

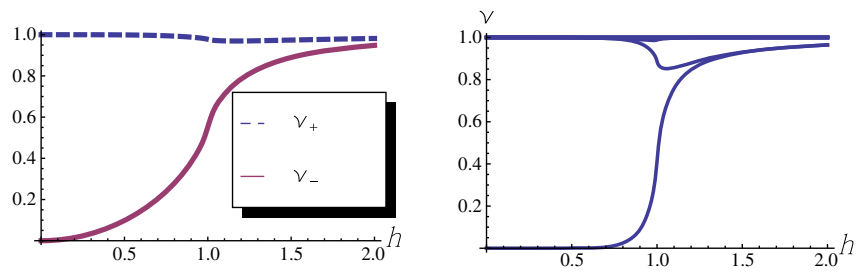

FIG. 2. Plot of the occupation number $\nu_{j}$ obtained from the correlation matrix (A6) as a function of $h$ for $L=2$ (left) and $L=10$ (right). For $L=2$, the explicit form of the eigenvalues $\nu_{ \pm}$ is given in Eq. (A8). Notice that only one of the $\nu$ 's exhibits nontrivial behavior: It corresponds to the boundary state, which is only partially contained in the subregion.

occupation number of this edge excitation increases, indicating edge-state recombination.

Having discussed the behavior of the eigenvalues $\nu_{j}$ 's and the role of the boundary states, it is straightforward to analyze the Rényi entropy and address the issue of differential local convertibility. It is interesting to concentrate on the two extreme limits: $L=2$ and $L \rightarrow \infty$.

The two occupation numbers $\nu_{ \pm}$for $L=2$ are shown in the left panel of Fig. 2, and the resulting four eigenvalues of the reduced density matrix, according to Eq. (A4), are plotted in the left panel of Fig. 3. While in locally convertible phases the largest eigenvalue(s) decrease approaching the QPT, indicating an increase of the entanglement, here we see that the edge-state recombination results in a growing larger eigenvalue. The right panel of Fig. 3 presents the sign of the entanglement entropy derivative, to be considered in relation with Eq. (2). We see that in the paramagnetic phase, the Rényi entropy always decreases. Instead, in the doubly degenerate phase, the entropy derivative vanishes at some value of $\alpha$ and changes sign, indicating that local (differential) convertibility is lost in this phase (as already observed numerically for small $N$ and larger $L$ in Ref. [32]). It is important to notice here that these results imply that any operation acting on two sites alone effectively creates edge states at the boundary of the sites and hence projects the system onto these states, losing part of the coherence in the original state.
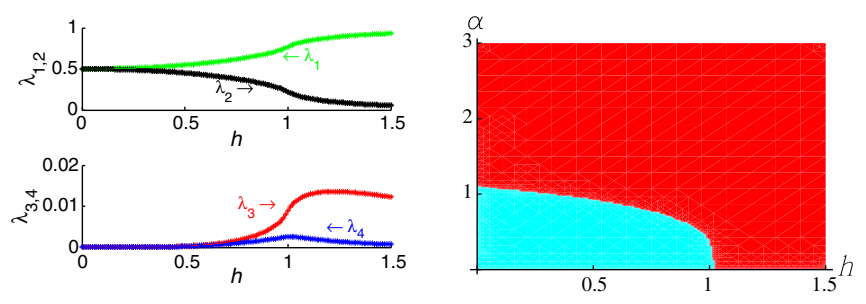

FIG. 3. Left: Plot of the four eigenvalues of $\rho_{A}$ for $L=2$, as a function of $h$. The solid lines depict analytical results, while the crosses show the numerical results with $N=200$. (Notice the different scales in the vertical axis between the top and bottom panels.) Right: Contour plot of the sign of the derivative with respect to $h$ of the Rényi entropy for different values of $h$ and $\alpha$.
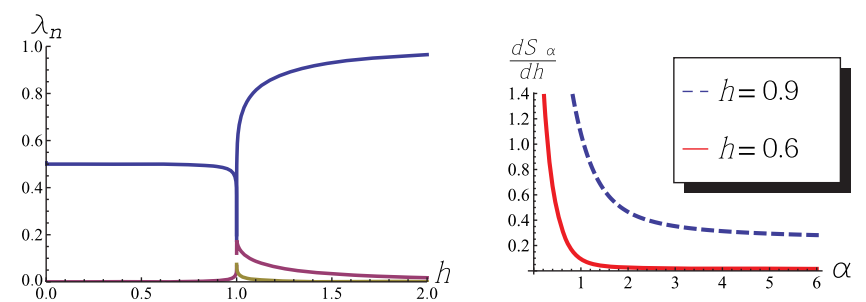

FIG. 4. Left: Plot of the first few eigenvalues of $\rho_{A}$, for an infinite size block, as a function of $h$. The eigenvalues' multiplicities are not shown. (For instance, the highest eigenvalue is doubly degenerate for $h<1$ and unique for $h>1$; see Ref. [44].) Right: Plot of the derivative of the Rényi entropy with respect to the magnetic field $h$, as a function of $\alpha$, for two different values of $h$ in the ferromagnetic region.

For the $L \rightarrow \infty$ limit, we can take advantage of the results of Refs. [44-46], where the full spectrum (eigenvalues and multiplicities) of the reduced density matrix and the Rényi entropies were calculated analytically. Figure 4 shows a plot of the first few eigenvalues of $\rho_{A}$ and a plot of the entropy derivative as a function of $\alpha$ for $h=0.6$ and $h=0.9$. We see that the largest eigenvalue (doubly degenerate in the ferromagnetic phase) decreases monotonously toward the QPT while smaller eigenvalues are allowed to grow, yielding a monotonous increase of all the Rényi entropies. It is thus clear that local convertibility is restored in the infinite $L$ limit.

We check these results numerically for systems up to $N=200$ and with different partitions. We consider different block sizes and move the blocks within the chain. The qualitative picture does not change significantly as one varies $(A \mid B)$, but the location of the curve where the entropy derivative vanishes transitions in the $(h, \alpha)$ plane. It tends toward the phase-transition line $h=1$ as the block sizes grow bigger, confirming our expectation on the role of the boundary excitations. Namely, we see that as long as the edge states from different boundaries do not overlap, their occupation number stays constant and vanishing. It starts increasing only once the correlation length grows comparable to one of the block sizes, indicating the recombination of the edge states and a decrease in the entanglement contribution from the edge states.

\section{SYMMETRY-BROKEN GROUND STATE}

To further confirm our interpretation on the role of boundary modes, in the ordered phase $h<1$, we also consider the ferromagnetic ground state for which $\left\langle\sigma^{x}\right\rangle \neq 0$. Since this state does not support well-defined Majorana edge states, we expect a restoration of local convertibility. We numerically calculate the Rényi entropy of this symmetry-broken ground state. Namely, we add a very small perturbation $\epsilon\left(\sigma_{1}^{x}+\sigma_{N}^{x}\right)$ to the Hamiltonian (3) and apply the variational matrix-product state routine to obtain 

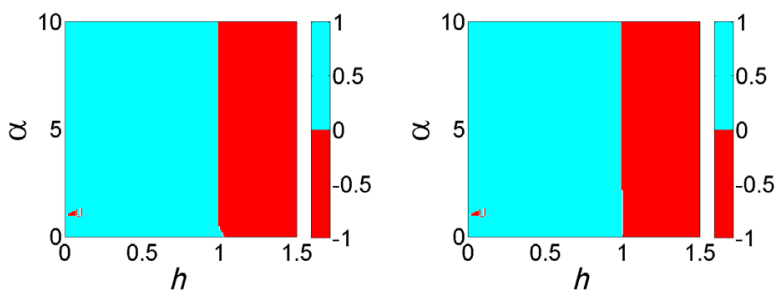

FIG. 5. Numeric results of differential local convertibility for the ferromagnetic (symmetry broken). Left: A partition $200=2 \mid 198$. Right: $200=50|100| 50$.

the ground state [47]. In this work, the convergence tolerance is $10^{-6}$. Figure 5 shows the plots of the sign of the entropy derivative for two possible partitions (small and large $A$ blocks), and it validates our expectation that both phases are locally convertible. We consider several partitioning choices, and the results are not distinguishable from those in Fig. 5.

In conclusion, we see that for $h>1$, the disordered ground state is always locally convertible. In the ordered phase, the ferromagnetic ground state, i.e., with broken symmetry, is also locally convertible for any chosen partition. For the thermal ground state, however, the convertibility depends on the interplay between the size of the partitions $(A \mid B)$ and the correlation length of the system. This phenomenon is a manifestation of edge-state recombination. These entangled pairs lie on opposite boundaries of the partition (see Fig. 1) but with a finite support intruding in the bulk about the order of the correlation length. For sufficiently large block size, the entanglement between boundary states does not depend on the correlation length and remains constant throughout the phase. However, as this length increases approaching a QPT, the edge states effectively grow closer. If either of the subregions $A$ or $B$ is sufficiently small, the tails of these states can overlap and we see their occupation number increasing and their entanglement decreasing, yielding lack of local convertibility.

\section{CONCLUSIONS AND DISCUSSIONS}

We considered a specific notion of long-range entanglement, defined as the lack of differential local convertibility for a bipartite system $(B|A| B)$. In phases where differential local convertibility holds, the response of the ground state to an external perturbation can be rendered by local means (in $A$ and $B$ ), and hence, such phases offer more restricted computational capability under adiabatic perturbation. By identifying a mechanism that breaks differential local convertibility, we discussed the role of edge states in determining quantitatively whether the adiabatic perturbation of a quantum many-body system can supersede a protocol limited to LOCC operations between $A$ and $B$.

In the class of models we considered, we found that destroying edge states (for instance, by breaking the symmetry of the Hamiltonian) yields locally convertible quantum phases. In contrast, in quantum phases with edge states, the convertibility depends on the interplay between the sizes of the partitions and on the correlation length of the system. Approaching the QPT, at some point, the correlation length grows to be comparable with one of the block sizes and the boundary states start recombining: From this point forward, their contribution to the entanglement starts decreasing. For sufficiently large $\alpha$ 's, the Rényi entropies are dominated by the edge-state behavior and they decrease toward a QPT. At the same time, low- $\alpha$ entropies still increase as usual because more states are required to construct the reduced density matrix in the Schmidt decomposition [26]. This combined behavior leads to a breakdown of local convertibility. To summarize, the entanglement of bulk states increases toward a QPT while that of edge states does the opposite. Since classical manipulations can never increase entanglement, this contrasting behavior signals the existence of genuine quantum character in these states. We remark that in LOCC, "local" means that the manipulations are restricted within each region of the bipartition and thus can still involve entangling operations, while in physical applications, locality has a much stricter meaning. For this reason, we believe that our observation that the edge-state recombination scheme works already for the smallest partitions $L=2$ (Fig. 3) has an important implication: The construction of quantum circuits already in terms of two-qubit gates generates a long-range coherence (between the Majorana fermions at the edges of the block) that, if incorporated, may provide an added value for the computation in the quantum phase. The two quantum phases possess qualitatively different computational capabilities: Indeed, it was shown in Ref. [48] that to connect them, one needs a quantum circuit whose depth scales at least linearly with the system's size; in contrast, different ground states within the same quantum phase can be connected with a depth sublinear in $N[48,49]$. As a step forward in this scenario, we identify in the local convertibility the "bit" providing the qualitative difference in resources between the two quantum phases.

Because of the fundamental relation between quantum circuits and Hamiltonian satisfiability problems [5,6], we believe that our results are relevant in the framework of the theory of quantum complexity classes [4,50-52]. Indeed, universal quantum computers encompass Hamiltonian models within the QMA complete complexity class. Notwithstanding, the quantum Ising model we considered in the present article is believed to be QMA complete only if it is suitably generalized [39,40,53]; our results indicate that the edge states should survive in these more general models, and their convertibility properties will be investigated in the future. It is also interesting to understand whether these more general models can be reached adiabatically starting from the quantum Ising chain, without crossing a QPT. 
Incidentally, we comment that a similar lack of differential local convertibility has been observed recently in topologically ordered systems [54,55]. In Ref. [54], in particular, it was demonstrated how the phase with nontrivial edge states, the Haldane phase, is indeed not locally convertible; given the role of the Haldane order in systems of cold atoms with dipolar interaction, this observation opens the avenue toward experimental verifications of the convertibility protocols. Indeed, topological systems are characterized by a form of long-range entanglement, as a finite-depth quantum circuit can disentangle the system only at short range $[49,56]$. Compared to this definition, we remark that local convertibility seems to detect a form of long-range entanglement, even while working on relatively small systems. We believe further research is called for, to understand the exact relation between local convertibility and the form of long-range entanglement, as defined in Ref. [49].

To conclude, the universal quantum simulator, as envisioned by Feynman [1], was thought for simulating all quantum interactions, hence superseding in efficiency any classical algorithm. Our theory shows that edge states provide phases that are not locally convertible, and this result indicates that a quantum simulator should show similar nonconvertibility. In this respect, it is not accidental that protected edge states play a crucial role in quantum computation (although it is clear that the existence of edge states is only a necessary condition for a system to be viable as a universal quantum simulator) [57]. This observation should direct further efforts toward the identification of workable quantum simulators and could open a way for a next generation of specifically designed quantum algorithms.

\section{ACKNOWLEDGMENTS}

We thank Alioscia Hamma for several fruitful discussions. F. F. thanks Chen Fang for his interesting seminar and his insights. F. F. was supported by a Marie Curie International Outgoing Fellowship within the 7th European Community Framework Programme (No. FP7/2007-2013) under Grant No. PIOF-PHY-276093. J. C. acknowledges Max-Planck-Gesellschaft Rechenzentrum Garching for the computational resource and thanks Mari Carmen Bañuls for discussions on numerical simulation. H. F. is supported by the 973 Program (No. 2010CB922904) and Grants from the NSFC and CAS. V. K. is supported by the NSF Grant No. DMS-1205422. L. C. K. and V. V. are supported by the National Research Foundation \& Ministry of Education, Singapore. M. G. is supported by the National Basic Research Program of China Grants No. 2011CBA00300 and No. 2011CBA00302 and by the National Natural Science Foundation of China Grants No. 11450110058, No. 61033001, and No. 61061130540. L. C. K. and L. A. are also supported by the National Institute of Education and the Institute of Advanced Studies, Nanyang Technological University.

\section{APPENDIX METHODS}

\section{The Rényi entropies}

An advantage of working with a quadratic theory such as the Ising chain is that many-body states can be constructed exactly out of individual quasiparticle excitations. The latter can be found as the linear combination of the fermionic operators $\left\{c_{j}, c_{j}^{\dagger}\right\}$ in Eq. (4), which diagonalize the Hamiltonian. Doing so, we define a new set of operators $\left\{\tilde{c}_{j}, \tilde{c}_{j}^{\dagger}\right\}$ so that the ground state $|g\rangle$ is annihilated by all $\tilde{c}_{j}$. Additionally, one can excite quasiparticles by progressively applying all possible combinations of $\tilde{c}_{j}^{\dagger}$, yielding a total of $2^{N}$ states in the Hilbert space.

To calculate the entanglement between the subregions $A$ and $B$, we employ the Schmidt decomposition of the ground state

$$
|g\rangle=\sum_{l} \sqrt{\lambda_{l}}\left|\psi_{l}^{(A)}\right\rangle\left|\psi_{l}^{(B)}\right\rangle,
$$

where $\left|\psi_{l}^{(A, B)}\right\rangle$ span the Hilbert space of blocks $A$ and $B$, respectively [26]. We are after the eigenvalues $\lambda_{l}$, which can be found, for instance, as

$$
\lambda_{l}=\left\langle g \mid \psi_{l}^{(A)}\right\rangle\left\langle\psi_{l}^{(A)} \mid g\right\rangle,
$$

where a trace over the $B$ degrees of freedom is implicit. Similarly to what is done for the entire system, the states $\left|\psi_{l}^{(A)}\right\rangle$ can be constructed in terms of individual excitations. However, these excitations differ from those of the whole chain, as they are contained inside the block. If $A$ consists of $L$ consecutive sites, these block excitations $\left\{d_{j}, d_{j}^{\dagger}\right\}$ are the linear combinations of the $c$ operators within the block that diagonalize the correlation matrix constructed out of all their two-point correlation functions, as shown below. Each state $\left|\psi_{l}^{(A)}\right\rangle$ of this $2^{L}$-dimensional Hilbert space can thus be characterized by the occupation number 0 or 1 of each block excitation. Moreover, the eigenvalues $\nu_{j}$ of the aforementioned correlation matrix provide us with the expectation values

$$
\left\langle g\left|d_{j} d_{j}^{\dagger}\right| g\right\rangle=\frac{1+\nu_{j}}{2}, \quad\left\langle g\left|d_{j}^{\dagger} d_{j}\right| g\right\rangle=\frac{1-\nu_{j}}{2},
$$

with all other correlations being 0 . Note that $\nu_{j} \simeq 1$ indicates that $d_{j}$ annihilates the vacuum $|g\rangle$. It follows that certain quasiparticle excitations of the Hamiltonian are completely contained within the block, since $d_{j}|g\rangle=0$ implies that $d_{j}$ is just a superposition of $\tilde{c}_{j}$ 's. Since $d_{j}$ is defined just within the block, it follows that these $\tilde{c}_{j}$ 's are also contained in the block. Conversely, smaller values of $\nu_{j}$ are related to excitations lying only partially within a subregion. In turn, $d_{j} d_{j}^{\dagger}$ acts on the ground state as a projection operator that selects the component with 0 
occupation number for the $l$ th block excitation while $d_{j}^{\dagger} d_{j}$ projects it onto an occupied $l$ th excitation. Hence, Eq. (A2) can be written as the expectation value of a string of operators of this type. Using Eq. (A3) as the building blocks of these correlators, we have

$$
\left\{\lambda_{l}\right\}=\prod_{j=1}^{L}\left(\frac{1 \pm \nu_{j}}{2}\right),
$$

with all the possible combinations of plus or minus signs, corresponding to the occupation or unoccupation of the different block excitations.

Finally, the Rényi entropies read [41,42]

$$
S_{\alpha}\left(\rho_{A}\right)=\frac{1}{1-\alpha} \sum_{j=1}^{L} \log \left[\left(\frac{1+\nu_{j}}{2}\right)^{\alpha}+\left(\frac{1-\nu_{j}}{2}\right)^{\alpha}\right] \text {. }
$$

\section{The correlation matrix}

The Rényi entropies are accessed through the eigenvalues of the reduced density matrix of a block of $L$ consecutive spins for the thermal ground state [41,42]. Such eigenvalues can be obtained from the diagonalization of the $2 L \times 2 L$ correlation matrix: $\left\langle f_{k}^{(a)} f_{j}^{(b)}\right\rangle=$ $\delta_{j, k} \delta_{a, b}+i\left(\mathcal{B}_{L}\right)_{(j, k)}^{(a, b)}$, with

$$
\mathcal{B}_{L} \equiv\left(\begin{array}{cccc}
\Pi_{0} & \Pi_{1} & \ldots & \Pi_{L-1} \\
\Pi_{-1} & \Pi_{0} & & \vdots \\
\vdots & & \ddots & \vdots \\
\Pi_{1-L} & \ldots & \ldots & \Pi_{0}
\end{array}\right),
$$

where $j, k$ specifies the entry

$$
\Pi_{j-k} \equiv\left(\begin{array}{cc}
0 & g_{j-k} \\
-g_{k-j} & 0
\end{array}\right)
$$

which is itself a $2 \times 2$ matrix whose $a, b$ entries are defined as

$$
g_{j} \equiv \frac{1}{2 \pi} \int_{0}^{2 \pi} \frac{\cos \theta-h+i \sin \theta}{\sqrt{(\cos \theta-h)^{2}+\sin ^{2} \theta}} e^{i j \theta} d \theta .
$$

The antisymmetric matrix $\mathcal{B}$ can be brought into a block-diagonal form by an $S O(2 L)$ rotation, with each block of the form

$$
\tilde{\Pi}_{j}=\nu_{j}\left(\begin{array}{cc}
0 & 1 \\
-1 & 0
\end{array}\right) .
$$

This rotation defines a new set of Majorana fermions $\tilde{f}_{j}^{(a)}$ with only pairwise correlations. This rotated-operator basis can be used to introduce a new set of complex operators: $d_{j}=\left(\tilde{f}_{j}^{(1)}+i \tilde{f}_{j}^{(2)}\right) / 2$ (and its Hermitian conjugate). The matrix (A6) contains all information to completely solve the model. By taking $L=N$, i.e., extending the correlation matrix to the whole system, the $d$ modes coincide with the $\tilde{c}$ operators one would obtain from the diagonalization of the Hamiltonian (3).

For $L=2$, the two eigenvalues of the correlation matrix are easily found to be

$$
\nu_{ \pm}=\sqrt{\left(\frac{g_{1}-g_{-1}}{2}\right)^{2}+g_{0}^{2}} \pm \frac{g_{1}+g_{-1}}{2},
$$

which allows for a complete analytical study of the entanglement entropy and its derivative (see Fig. 3).

[1] R. P. Feynman, Simulating Physics with Computers, Int. J. Theor. Phys. 21, 467 (1982).

[2] S. Lloyd, Universal Quantum Simulators, Science 273, 1073 (1996).

[3] E. Knill, R. Laflamme, R. Martinez, and C.-H. Tseng, An Algorithmic Benchmark for Quantum Information Processing, Nature (London) 404, 368 (2000).

[4] E. Bernstein and U. Vazirani, Quantum Complexity Theory, SIAM J. Comput. 26, 1411 (1997).

[5] A. Kitaev, A. Shen, and M. Vyalyi, Classical and Quantum Computation, Graduate Studies in Mathematics Vol. 47 (AMS, Providence, RI, 2002).

[6] D. Aharonov, W. van Dam, J. Kempe, Z. Landau, S. Lloyd, and O. Regev, Adiabatic Quantum Computation Is Equivalent to Standard Quantum Computation, SIAM J. Comput. 37, 166 (2007).

[7] J. D. Whitfield, J. Biamonte, and A. Aspuru-Guzik, Simulation of Electronic Structure Hamiltonians Using Quantum Computers, Mol. Phys. 109, 735 (2011).

[8] I. Kassal, J. D. Whitfield, A. Perdomo-Ortiz, M. H. Yung, and A. Aspuru-Guzik, Simulating Chemistry Using Quantum Computers, Annu. Rev. Phys. Chem. 62, 185 (2011).

[9] L. Amico, R. Fazio, A. Osterloh, and V. Vedral, Entanglement in Many-Body Systems, Rev. Mod. Phys. 80, 517 (2008).

[10] J. Eisert, M. Cramer, and M. B. Plenio, Area Laws for the Entanglement Entropy, Rev. Mod. Phys. 82, 277 (2010).

[11] J. I. Cirac and P. Zoller, Goals and Opportunities in Quantum Simulation, Nat. Phys. 8, 264 (2012).

[12] I. Bloch, J. Dalibard, and S. Nascimbene, Quantum Simulations with Ultracold Quantum Gases, Nat. Phys. 8, 267 (2012).

[13] A. Aspuru-Guzik and P. Walther, Photonic Quantum Simulators, Nat. Phys. 8, 285 (2012).

[14] A. A. Houck, H. E. Tureci, and J. Koch, On-Chip Quantum Simulation with Superconducting Circuits, Nat. Phys. 8, 292 (2012).

[15] L. M. K. Vandersypenm and I. J. Chuang, NMR Techniques for Quantum Control and Computation, Rev. Mod. Phys. 76, 1037 (2005).

[16] G. Vidal, Efficient Classical Simulation of Slightly Entangled Quantum Computations, Phys. Rev. Lett. 91, 147902 (2003).

[17] M. Van den Nest, W. Dür, G. Vidal, and H. J. Briegel, Classical Simulation Versus Universality in Measurement-Based Quantum Computation, Phys. Rev. A 75, 012337 (2007). 
[18] M. Van den Nest, W. Dür, and H. J. Briegel, Completeness of the Classical 2D Ising Model and Universal Quantum Computation, Phys. Rev. Lett. 100, 110501 (2008).

[19] M. J. Bremner, C. Mora, and A. Winter, Are Random Pure States Useful for Quantum Computation?, Phys. Rev. Lett. 102, 190502 (2009).

[20] D. Gross, S. T. Flammia, and J. Eisert, Most Quantum States Are Too Entangled to Be Useful as Computational Resources, Phys. Rev. Lett. 102, 190501 (2009).

[21] G. De las Cuevas, W. Dür, M. Van den Nest, and H. J. Briegel, Completeness of Classical Spin Models and Universal Quantum Computation, J. Stat. Mech. (2009) P07001.

[22] M. Van den Nest, Universal Quantum Computation with Little Entanglement, Phys. Rev. Lett. 110, 060504 (2013).

[23] R. Raussendorf, Contextuality in Measurement-Based Quantum Computation, Phys. Rev. A 88, 022322 (2013).

[24] D. Gottesman, Stabilizer Codes and Quantum Error Correction, arXiv:quant-ph/9705052.

[25] Z. C. Gu and X. G. Wen, Tensor-Entanglement-Filtering Renormalization Approach and Symmetry-Protected Topological Order, Phys. Rev. B 80, 155131 (2009).

[26] M. A. Nielsen and I. L. Chuang, Quantum Computation and Quantum Information (Cambridge University Press, Cambridge, England, 2004).

[27] M. A. Nielsen, Conditions for a Class of Entanglement Transformations, Phys. Rev. Lett. 83, 436 (1999).

[28] D. Jonathan and M. B. Plenio, Entanglement-Assisted Local Manipulation of Pure Quantum States, Phys. Rev. Lett. 83, 3566 (1999).

[29] See Supplemental Material at http://link.aps.org/ supplemental/10.1103/PhysRevX.4.041028 for an example of the connection between local convertibility and majorization of the Renyi entropies.

[30] S. Turgut, Catalytic Transformations for Bipartite Pure States, J. Phys. A 40, 12185 (2007).

[31] M. Klimesh, Inequalities That Collectively Completely Characterize the Catalytic Majorization Relation, arXiv:0709.3680.

[32] J. Cui, M. Gu, L. C. Kwek, M. F. Santos, H. Fan, and V. Vedral, Quantum Phases with Differing Computational Power, Nat. Commun. 3, 812 (2012).

[33] E. Farhi, J. Goldstone, S. Gutmann, J. Lapan, A. Lundgren, and D. Preda, A Quantum Adiabatic Evolution Algorithm Applied to Random Instances of an NP-Complete Problem, Science 292, 472 (2001).

[34] M. Z. Hasan and C. L. Kane, Topological Insulators, Rev. Mod. Phys. 82, 3045 (2010).

[35] V. Mourik, K. Zuo, S. M. Frolov, S. R. Plissard, E. P. A. M. Bakkers, and L. P. Kouwenhoven, Signatures of Majorana Fermions in Hybrid Superconductor-Semiconductor Nanowire Devices, Science 336, 1003 (2012).

[36] E. Lieb, T. Schultz, and D. Mattis, Two Soluble Models of an Antiferromagnetic Chain, Ann. Phys. (N.Y.) 16, 407 (1961).

[37] E. Barouch and B. M. McCoy, Statistical Mechanics of the XY Model. II. Spin-Correlation Functions, Phys. Rev. A 3, 786 (1971).

[38] R. Coldea, D. A. Tennant, E. M. Wheeler, E. Wawrzynska, D. Prabhakaran, M. Telling, K. Habicht, P. Smeibidl, and K. Kiefer, Quantum Criticality in an Ising Chain:
Experimental Evidence for Emergent $E_{8}$ Symmetry, Science 327, 177 (2010).

[39] J. D. Biamonte and P. J. Love, Realizable Hamiltonians for Universal Adiabatic Quantum Computers, Phys. Rev. A 78, 012352 (2008).

[40] R. Oliveira and B. M. Terhal, The Complexity of Quantum Spin Systems on a Two-Dimensional Square Lattice, Quantum Inf. Comput. 8, 0900 (2008).

[41] G. Vidal, J. I. Latorre, E. Rico, and A. Kitaev, Entanglement in Quantum Critical Phenomena, Phys. Rev. Lett. 90, 227902 (2003).

[42] J. I. Latorre, E. Rico, and G. Vidal, Ground State Entanglement in Quantum Spin Chains, Quantum Inf. Comput. 4, 48 (2004).

[43] A. Kitaev, Unpaired Majorana Fermions in Quantum Wires, Phys. Usp. 44, 131 (2001).

[44] F. Franchini, A. R. Its, V. E. Korepin, and L. A. Takhtajan, Spectrum of the Density Matrix of a Large Block of Spins of the XY Model in One Dimension, Quantum Inf. Process. 10, 325 (2011).

[45] A. R. Its, B.-Q. Jin, and V. E. Korepin, Entanglement in the XY Spin Chain, J. Phys. A 38, 2975 (2005).

[46] F. Franchini, A. R. Its, and V. E. Korepin, Rényi Entropy of the XY Spin Chain, J. Phys. A 41, 025302 (2008).

[47] F. Verstraete, V. Murg, and J. I. Cirac, Matrix Product States, Projected Entangled Pair States, and Variational Renormalization Group Methods for Quantum Spin Systems, Adv. Phys. 57, 143 (2008).

[48] Y. Huang and X. Chen, Quantum Circuit Complexity of One Dimensional Topological Phases, arXiv:1401.3820.

[49] X. Chen, Z.-C. Gu, and X.-G. Wen, Local Unitary Transformation, Long-Range Quantum Entanglement, Wave Function Renormalization, and Topological Order, Phys. Rev. B 82, 155138 (2010).

[50] J. Kempe, A. Kitaev, and O. Regev, The Complexity of the Local Hamiltonian Problem, SIAM J. Comput. 35, 1070 (2006).

[51] S. Bravyi, D. P. DiVincenzo, R. I. Oliveira, and B. M. Terhal, The Complexity of Stoquastic Local Hamiltonian Problems, Quantum Inf. Comput. 8, 0361 (2008).

[52] M. Gu and A. Perales, Encoding Universal Computation in the Ground States of Ising Lattices, Phys. Rev. E 86, 011116 (2012).

[53] D. Gottesman and S. Irani, The Quantum and Classical Complexity of Translationally Invariant Tiling and Hamiltonian Problems, Theory Comput. 9, 31 (2013).

[54] J. Cui, L. Amico, H. Fan, M. Gu, A. Hamma, and V. Vedral, Local Characterization of One-Dimensional Topologically Ordered States, Phys. Rev. B 88, 125117 (2013).

[55] A. Hamma, L. Cincio, S. Santra, P. Zanardi, and L. Amico, Local Response of Topological Order to an External Perturbation, Phys. Rev. Lett. 110, 210602 (2013).

[56] M. B. Hastings and X.-G. Wen, Quasi-adiabatic Continuation of Quantum States: The Stability of Topological Ground State Degeneracy and Emergent Gauge Invariance, Phys. Rev. B 72, 045141 (2005).

[57] C. Nayak, S. H. Simon, A. Stern, M. Freedman, and S. Das Sarma, Non-Abelian Anyons and Topological Quantum Computation, Rev. Mod. Phys. 80, 1083 (2008). 